\title{
Cancer researchers usher in dog days of medicine
}

After thousands of years of friendship, man and dog will soon be working together to advance drug discovery. As part of a new comparative-oncology program at the US National Cancer Institute, researchers are set to begin drug trials in dogs that aim to bridge ๖ the gap between preclinical drug studies and effects of the same drug in people.

Two multicenter trials, set to begin in early 2006, will evaluate new therapeutic approaches for cancer in dogs, says program chief Chand Khanna, a veterinary oncologist at the institute's Center for Cancer Research.

"It turns out that many cancers, such as lymphoma, melanoma and osteosarcoma, are well represented in pet dogs," Khanna says. "The dogs will help us prioritize the agents that go into human clinical trials and help make those trials more efficient."

The idea of testing drugs in canines is not new. As far back as 1922, Frederick Banting and Charles Best treated a critically ill 14-year-old diabetic with insulin after first experimenting with dogs at the University of Toronto.

Much of cancer research relies on rodent models, which help answer specific biological questions. "But they have several limitations," says oncologist David Vail of Colorado State University's Animal Cancer Center. Vail notes that dogs' body size allows for easier collection of samples such as serum and urine. Surgical intervention and imaging in dogs also more closely replicate the human clinical setting.

The kinship extends into the molecular realm. For instance, enzyme systems and kinetics within the cytochrome $\mathrm{P} 450$ pathway in humans is more similar in dogs than in rodents. Researchers can measure the enzymes' effect on known substrates, note metabolic endpoints and measure the speed with which reactions occur in different species. "The rate at which dogs metabolize and clear drugs," says Vail, "is more similar to humans than that of rodents."

Now that the dog's genome has been mapped, the venerable creature is better positioned to advance disease understanding. But there's another factor that makes it a good experimental model: it has shared a common environment with humans for thousands of years.

Companion dogs represent a more natural population than inbred laboratory animals. "Outbred dogs with spontaneously arising tumors more closely mimic the variability of tumor biology and response to treatment that is seen in people with cancer," says Vail.

Elsewhere, scientists are using dogs in other

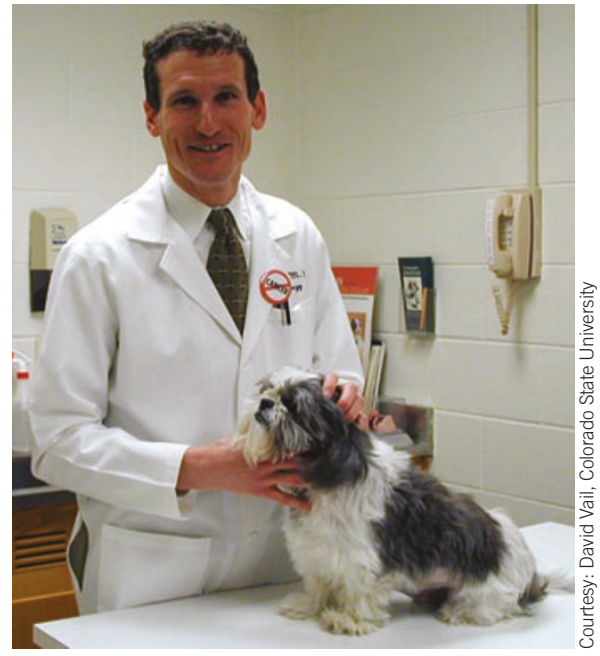

Oncologist David Vail says dogs mimic human tumors more closely than do rodents.

research areas such as spinal injury. Richard Borgens, director of the Veterinary Center for Paralysis Research at Purdue University, and his colleagues have injected liquid polymer polyethylene glycol into the injured spines of paralyzed dogs. Injected within 72 hours of injury, 13 of the 19 dogs regained use of their hind legs and were able to walk (J. Neurotrauma 21, 1767-1777; 2004).

Borgens says some of the dogs recovered well enough to carry on as if they had never been injured. Based on these data and previous work on guinea pig spinal cords, the method will be tested in 2006 in trials for acute spinal injury.
Another team of researchers at the University of Pennsylvania is testing dogs in gene therapy studies that use an adeno-associated virus vector to treat hereditary eye diseases such as retinitis pigmentosa, including an early-onset form that causes blindness at birth. In human trials, the researchers would inject the vector under the retina in the macula region where central vision is sensed, but in dogs treat a homologous site known as the area centralis.

Typical laboratory animals, especially rodents, are nocturnal creatures, and don't rely much on their vision for normal everyday behavior. "But the dog is a wonderful disease model," says molecular geneticist Jean Bennett. "They are visually responsive, and when you treat the dog there is such an intuitive understanding of the remarkable effect of restoring vision.” Bennett says a phase 1 human gene therapy trial will begin at Penn by mid-2006.

The closeness between dogs and people, much as it facilitates research, also presents an ethical conundrum. And researchers who work with dogs are more likely to be a target for animal rights activists.

But the researchers say drugs should first be tested at increasing doses in rodents before being tested in dogs, and the results are often beneficial for the animals themselves. "There is an eye towards the flow of information being bidirectional and coming back to the veterinary patient," says Vail. "At the end of the day, I'm a veterinarian, and I want to help companion animals."

George S Mack, Columbia, South Carolina

\section{NIH rolls back financial conflict rules}

After seven tense months, the US National Institutes of Health (NIH) on 25 August rolled back its controversial 'interim final rule,' designed to prohibit conflicts of interest among its researchers.

Director Elias Zerhouni announced that the total ban on NIH researchers consulting with pharmaceutical and related industries would remain in force. However, only the institute's 200 or so senior employees will be required to divest stocks from those companies in excess of $\$ 15,000$, whereas all employees would still be scrutinized for conflicts.

But in some cases, it is "still unclear" what the new rules at the NIH would allow, says Derek LeRoith who in September left the NIH for New York's Mount Sinai School of Medicine (Nat. Med. 11, 914-915; 2005). LeRoith says he's happy with his decision to leave the agency after 26 years there because it allows him to continue as president of the International Society for IGF Research, to lecture "and get reimbursed for my efforts."

Since newspaper reports of NIH scientists violating the agency's conflict of interest guidelines first appeared in December 2003, $\mathrm{NIH}$ policies have been the focus of intense debate. Zerhouni said the agency would continue to insist on full disclosure and consider employees' holdings on an individual basis. Some employees still must file public forms, and can only accept awards with prior approval from an ethics officer.

George S Mack, Columbia, South Carolina

For more news and analysis go to news@nature.com www.nature.com/news 